\title{
Karl Kraus et le naturalisme
}

Karl Kraus und der Naturalismus

Karl Kraus and naturalism

Jacques Le Rider

\section{OpenEdition}

\section{Journals}

Édition électronique

URL : http://journals.openedition.org/austriaca/567

DOI : 10.4000/austriaca.567

ISSN : 2729-0603

\section{Éditeur}

Presses universitaires de Rouen et du Havre

\section{Édition imprimée}

Date de publication : 1 juin 2018

Pagination : 99-116

ISBN : 979-10-240-1233-9

ISSN : 0396-4590

\section{Référence électronique}

Jacques Le Rider, « Karl Kraus et le naturalisme », Austriaca [En ligne], 86 | 2018, mis en ligne le 01 juillet 2020, consulté le 30 janvier 2021. URL : http://journals.openedition.org/austriaca/567 ; DOI : https://doi.org/10.4000/austriaca.567 
Jacques Le RIDER

EPHE, Paris

\section{Karl Kraus et le naturalisme}

\section{Pour Gerhart Hauptmann}

Comme l'écrit Harry Zohn au début de son étude sur Karl Kraus et l'expressionnisme, Kraus avait les «-ismes » en horreur ${ }^{1}$. Il admirait Else Lasker-Schüler et Georg Trakl, mais ne se privait pas de critiquer l'expressionnisme. De même, on peut dire que son admiration, jamais reniée, pour le Gerhart Hauptmann des Tisserands (Die Weber) ${ }^{2}$ ne permet pas de parler d'une préférence que Kraus aurait eue pour le naturalisme, fût-il berlinois, dans lequel il aurait vu l'antidote au poison de l'esthétisme de la Jeune Vienne. En réalité, notre «entrepreneur de démolitions" (s'il est permis d'appliquer à Karl Kraus cette formule de Léon Bloy) s'en prendra au naturalisme avec autant de verve qu'au cercle des poètes du Café Griensteidl disparu ${ }^{3}$.

Dès 1890 , le lycéen Karl Kraus a lu avec enthousiasme le drame social Vor Sonnenaufgang (1889) de Gerhart Hauptmann ${ }^{4}$. La découverte enthousiaste des Tisserands date de 1892 : en avril (quelques semaines avant les épreuves du bac), Karl Kraus publie dans la Wiener LiteraturZeitung sa première critique consacrée à la pièce de Hauptmann dans la

1. Harry Zohn, «Karl Kraus und der Expressionismus », in Expressionismus in Österreich. Die Literatur und die Künste, Klaus Amann et Armin Wallas (dir.), Wien-KölnWeimar, Böhlau, «Literatur in der Geschichte. Geschichte in der Literatur, vol. 30 », 1994, p. 515-525, citation p. 515.

2. Voir Werner Kraft, "Gerhart Hauptmann", dans Das Ja des Neinsagers. Karl Kraus und seine geistige Welt, München, text + kritik, 1974, p. 147-157.

3. Voir Jacques Le Rider, "Naturalismus in bleu-blanc-rouge, Schwarz-Weiß-Rot und Schwarzgelb", dans Roland Innerhofer et Daniela Strigl (dir.), Sonderweg in Schwarzgelb? Auf der Suche nach einem österreichischen Naturalismus, Innsbruck, Studien Verlag, 2016, p. 33-56.

4. Edward Timms, Karl Kraus. Satiriker der Apokalypse, Max Looser et Michael Strand (trad.), Wien, Deuticke, 1995, p. 394 [trad. de Karl Kraus, Apocalyptic Satirist, New Haven, Yale University Press, 1986]. 
version en dialecte silésien De Waber, publiée à Berlin chez S. Fischer ${ }^{5}$. En 1892-1893, les contemporains peuvent supposer que Kraus est un émule viennois du naturalisme allemand, puisqu'il collabore à Die Gesellschaft, revue publiée à Leipzig par l'écrivain naturaliste Michael Georg Conrad, à laquelle contribuent Karl Bleibtreu, Max Halbe et Richard Dehmel.

C'est dans les pages de Die Gesellschaft que Karl Kraus déclare la guerre à Hermann Bahr dont il a trouvé ridiculement infatué «le dépassement du naturalisme»:

Der Tagschreiber, der als Litterat überhaupt nicht mehr ernst zu nehmen ist [...], von dem es sprichwörtlich geworden, dass er die "Richtungen" nur so aus dem Hemdärmel herausschüttele und jeden Tag eine zum "Überwinden" brauche, der große „Überwinderdes Naturalismus" - und - und des Symbolismus. [...] Er lernte die "Sensationen" und "Heimlichkeiten" des schönen, nervendurchtobten Paris kennen und finden, dass das "liebe Wienerisch" mit seinem „weichen, parfumierten Formen" auch echtes Pariser Fin-de-siècle atme - und aus war's; da ward ihm denn der Realismus zu gesund, zu deutsch; Deutschland thut man einfach ab, dachte er sich, verwindet man. [...] Ich wenigstens empfinde ihn als wunderliche Kombination von heimlichen Nerven und unheimlicher Arroganz ${ }^{6}$.

Aux yeux de Karl Kraus, un des aspects les plus irritants des chroniques littéraires de Hermann Bahr est sa manie moderniste de faire la théorie des «-ismes» pour donner substance à ce que Kraus considère comme une construction factice de nature journalistique, c'est-à-dire vide de véritable sens, et pour mettre en scène le « dépassement » de cette même construction. A contrario, on pourrait dire que, pour l'antimoderne Karl Kraus ${ }^{7}$, l'étiquetage d'une œuvre - naturaliste, symboliste... - n'a

5. "Gerhart Hauptmann: „De Waber“ („Die Weber“)», dans Karl Kraus, Frühe Schriften 1892-1900, Johannes J. Braakenburg (éd.), München, Kösel, 1979 (2 vol.), t. I, p. 9-12. Première publication dans la Wiener Literatur-Zeitung, $3^{\mathrm{e}}$ année, $\mathrm{n}^{\mathrm{o}} 4$, avril 1892, p. 19 et suiv.

6. Karl Kraus, «Zur Überwindung des Hermann Bahr», dans Frühe Schriften, op. cit., t. I, p. 103-114, citation p. 108 et suiv., et p. 114. Première publication dans Die Gesellschaft, $9^{\mathrm{e}}$ année, mai 1893, p. 627-636. Traduction française: «Pour dépasser Hermann Bahr», dans Hermann Bahr, Ce monsieur de Linz qui inventa Vienne, textes de Hermann Bahr, Karl Kraus, Hugo von Hofmannsthal, Jean Launay (trad. et éd.), Monaco, Éditions du Rocher, 2006, p. 189-206, citation p. 196-198 et 204.

7. «Karl Kraus' Paris und die Antimoderne», dans «Wien-Paris im Licht der Fackel von Karl Kraus", Anna-Katharina Gisbertz et Eva-Tabea Meineke (dir.), Études germaniques, $71^{\mathrm{e}}$ année, $\mathrm{n}^{\mathrm{o}}$ 3, juillet-septembre 2016, p. 319-326. 
aucune importance et que seules comptent les œuvres indépassables. Ce que dépasse Hermann Bahr, Karl Kraus le laisse passer sans lui accorder d'importance. Les Tisserands de Gerhart Hauptmann, en revanche, lui apparaissent d'emblée comme un classique du temps présent. Kraus aime aussi beaucoup Hanneles Himmelfahrt (1893) de Hauptmann, mais, contrairement à la majorité des critiques, il ne se demande pas si cette pièce, naturaliste au premier acte, verserait à la fin dans le symbolisme ou le néoromantisme. Kraus appréciera également Und Pippa tanzt! (1906) de Hauptmann, en tout cas le premier acte, une pièce qui n'a plus grand-chose à voir avec le naturalisme, mais qu'on hésite aussi à qualifier de symboliste, tant le terme semble, en 1906, appartenir à une période antérieure.

Dans La Littérature démolie, le pamphlet de 1897 dans lequel Karl Kraus rompt avec les modernes viennois (Hofmannsthal, Schnitzler, Beer-Hofmann, etc.), il n'est pas question du naturalisme, mais seulement de l'École du Nord, dont les modernes du midi de l'Allemagne ont voulu se démarquer. En somme, la différence entre le naturalisme allemand et la modernité viennoise est ici conçue comme une rivalité entre Berlin et Vienne :

Die moderne Bewegung, die vor einem Jahrzehnt vom Norden ausging, hat hier nur rein technische Veränderungen hervorgerufen. Von der inneren Wirkung neuen Styls, der das Stoffgebiet erweitern half und sociale Probleme ins Rollen brachte, ist unsere junge Kunst verschont geblieben, die geradezu in der Abkehr von den geistigen Kämpfen der Zeit ihr Heil sucht. Wenn Gedankenarmut in Stimmungen schwelgen will, muss das Wienerthum für die Farbe herhalten, und der Localpatriotismus erwacht zu neuem, sensitiverem Dasein $^{8}$.

Ce n'est donc pas leur "manque de naturalisme» que Karl Kraus reproche aux modernes viennois du Jung Wien, mais leur "pauvreté de pensée » qui s'épanche en Stimmungen (sentiments, impressions, états d'âme...). L'expression "die moderne Bewegung", par laquelle commence le paragraphe, a une tonalité plutôt moqueuse et dépréciative: Kraus n'arbitrera pas la compétition entre mouvements modernes, entre naturalistes berlinois et " post-naturalistes » viennois. On ne trouve pas sous sa plume de plaidoyer pour le naturalisme en général. Si certaines œuvres de Gerhart Hauptmann ont à ses yeux le rang de classiques

8. Karl Kraus, «Die demolirte Literatur » [texte de la 5 éd., 1899], dans Frühe Schriften, op. cit., t. II, p. 277-297, citation p. 284 et suiv. [La Littérature démolie, Yves Kobry (trad.), Paris, Rivages poche, 1993, p. 57.] 
contemporains, Karl Kraus rejette d'autres naturalistes avec autant de véhémence que les nouvelles gloires de la Jeune Vienne. Mais il rejette aussi les antinaturalistes du genre de Hermann Bahr qui, après avoir volé de fleur en fleur sur le Parnasse naturaliste berlinois et parisien, se pose désormais en maître du dépassement du naturalisme - sans dépasser pour autant ses propres limites. Plus tard, Karl Kraus observera avec le même scepticisme sarcastique les nouveaux expressionnistes qui se poseront en antinaturalistes et anti-impressionnistes ${ }^{9}$.

On peut considérer la fidélité de Karl Kraus au Gerhart Hauptmann de la première période comme tout à fait exceptionnelle dans la mesure où elle a résisté à l'épreuve de la première guerre mondiale, durant laquelle Hauptmann commet plus d'un texte nationaliste et belliciste, et même à celle de la mise au pas de la culture allemande, à partir de 1933, alors que Hauptmann se prête sans résistance à toutes les récupérations par les nazis, tout en affectant une attitude de retrait hautain.

Lors de sa lecture publique du 4 avril 1917, à Vienne, Kraus met au programme Judith et Holoferne de Nestroy et Hannele Matterns Himmelfahrt. Il ajoute cette précision dans le programme : "Die Vorführung dieser im neueren Deutschland einzigartigen Dichtung geschieht, um häufig vorkommenden Verwechslungen des Dichters Gerhart Hauptmann mit dem Kriegsdichter gleichen Namens zu begegnen ${ }^{10}$. » Durant les années 1920, Karl Kraus mettra souvent au programme ses textes préférés de Gerhart Hauptmann. Lors de sa lecture publique du 25 novembre 1932, à Vienne, il choisira une fois Les Tisserands et il ajoutera cette déclaration mémorable au programme :

Auf die Frage, was ich gegen die Erscheinungen habe, die ich angreife, antworte ich : eben sie, und immer war alles ausgesprochen, nichts bleibt dahinter. Wer es vermutet, ist ein Lump, wem die Beweise nicht genügen, den entlasse ich gern als Esel. Gegen George habe ich also, daß er schlechte Verse macht; gegen den Krieg habe ich den Krieg, gegen die Presse die Presse, gegen die Sozialdemokratie : eben sie. Gegen meine Anhänger, daß sie dumme Fragen stellen. [...] Eine große Meinungsverschiedenheit, die bald wird ausgetragen werden müssen, scheint in Bezug auf Shakespeare zu herrschen, dem ich anhänge. Was Hauptmann betrifft, so sei gesagt, daß der

9. "In der Dichtkunst... Abkehr vom Naturalismus", écrit Paul Ferdinand Schmidt dans «Die Expressionisten", Der Sturm, n ${ }^{\circ}$ 92, janvier 1912, p. 734-736, citation p. 736.

10. Die Fackel, n ${ }^{\circ} 457-461,10$ mai 1917, p. 63. 
Vortrag ausschließlich der Feier der revolutionären Jugend dieses Dichters gilt $^{11}$.

Dans la Troisième nuit de Walpurgis, Gerhart Hauptmann est dénoncé comme un des auteurs allemands qui ont fait allégeance au nazisme. Il peut à présent voir «d'un bon œil l'éventualité que le maçon de Mattern devienne président de Silésie ${ }^{12}{ }$. Le silence dans lequel se retire Gerhart Hauptmann, tout en restant membre des plus prestigieuses institutions littéraires patronnées par le régime nazi, est un geste bien insuffisant: "Wenn ,Hauptmann schweigt', so ist es immer noch besser, als wenn Großmann spricht; aber ein Schritt von dem Wege, der ihn mit dem Bereich solcher Offizia verbindet, war von ihm zu erwarten, damit er nicht offiziös erscheine ${ }^{13}$.»

Après ces jugements sévères, dans la Troisième nuit de Walpurgis (non publiée), Karl Kraus fait preuve de plus d'indulgence dans «Pourquoi la Torche ne parait pas » de juillet $1934^{14}$ :

Es könnte sogar zweifelhaft sein, ob nicht der Zoll alternden Intellekts an die Natur mit einem Bekenntnis bezahlt wurde, das zwar mit seinem Inhalt, doch nicht mit seiner Ehrlichkeit zu allem Jugendschaffen kontrastiert. Aber wenn unter den Jammergestalten der Welt Gerhart Hauptmanns deren Schöpfer als die einprägsamste zurückbleibt, so möchte man doch nicht dem Webergesellen gleichen, der vor dem Aussichtslosen die Axt ergreift und mit dem Ruf davonstürzt : „Ufgepaßt, jetzt komm ich!"Das wäre so ungefähr die Rolle, die einem zugemutet wird, während man selber „Polemik“, die das Ungeheure mit dem Anspruch auf Wirkung anginge, für „Vermessenheit" im wahren Sinne hält, für ein Beginnen des Größenwahns - innerhalb der nun einmal gültigen Dimensionen - : vielleicht ein Schaustück der Literatur, woran sich diejenigen ergötzen, die vom Grauen noch unberührt sind - doch aller Voraussicht nach Zutat der Pein für eben jene, denen es Trost oder Genugtuung bedeuten sollte.

En 1934, Karl Kraus considère non sans un reste de sympathie le vieux Hauptmann qui n'aurait, selon lui, rien perdu de la franchise qui caractérisait ses œuvres de jeunesse et dont le silence face au nouveau

11. Die Fackel, $\mathrm{n}^{\circ} 885-887$, décembre 1932, p. 18.

12. Karl Kraus, Troisième nuit de Walpurgis, Pierre Deshusses (trad.), Marseille, Agone, 2005, p. 281 (Karl Kraus, Dritte Walpurgisnacht, dans Schriften, Christian Wagenknecht éd., Francfort/Main, Suhrkamp, «Taschenbuch, 1322 », 1989, vol. 12, p. 112 : "gefaßt der Eventualität entgegensehen, daß der Maurer Mattern Oberpräsident von Schlesien wird.»

13. Karl Kraus, Dritte Walpurgisnacht, op. cit., p. 113.

14. «Warum die Fackel nicht erscheint », Die Fackel, n 890-905, fin juillet 1934, p. 69 et suiv. 
régime nazi exprimerait, comme celui de Kraus lui-même entre la fin décembre $1932\left(\mathrm{n}^{\circ} 885-887\right.$ de La Torche), octobre $1933\left(\mathrm{n}^{\circ} 888\right.$, réduit à quatre pages, avec le poème « Man frage nicht...») et juillet $1934\left(\mathrm{n}^{0} 889\right.$, «Nachrufe auf Karl Kraus»), le sentiment d'impuissance d'un auteur de textes littéraires face à la violence déchaînée de la dictature.

\section{Contre la manière naturaliste berlinoise}

L'attachement de Karl Kraus au Gerhart Hauptmann des années 1890 et du début du siècle suivant a donc résisté aux pires épreuves. Mais cet attachement ne peut pas être interprété comme une fidélité de Kraus au naturalisme. Dans les années 1890, avant le lancement de Die Fackel, Kraus prend le contrepied des options "anti-naturalistes" de Hermann Bahr et peut apparaître comme un partisan du naturalisme des acteurs et des metteurs en scène berlinois. Mais le ton change à partir de 1899. Dès la première année de Die Fackel, Karl Kraus multiplie les remarques sarcastiques à propos du naturalisme. En somme, Kraus apprécie Gerhart Hauptmann parce que le grand art de l'auteur des Tisserands lui a permis de transcender le maniérisme naturaliste, sans qu'il ait jamais cherché à « dépasser le naturalisme», selon la formule de Hermann Bahr que Kraus juge dérisoire.

Dès le numéro 3 de Die Fackel, fin avril 1899, Karl Kraus se moque d'une pièce d'Ernst Rosmer (pseudonyme d'Elsa Bernstein) représentée au Burgtheater qui montre comment le "naturalisme descriptif», sur scène, finit par se confondre avec le réalisme bourgeois confortable des comédies de boulevard d'Adolph L'Arronge, le prédécesseur d'Otto Brahm, le promoteur du naturalisme, à la tête du Deutsches Theater de Berlin :

Allerdings zeigt die Dichterin in geradezu erschreckend deutlicher Weise, wie der ganze schildernde Naturalismus, der in den Details einer philiströsen Häuslichkeit aufgeht, schließlich in L'Arronge'scher Gemütlichkeitsmeierei enden muss und sich nur Dank seiner schlechteren Mache als selbständiges Genre noch $z u$ behaupten vermag ${ }^{15}$.

Dans le numéro 10 de début juillet 1899, à l'occasion d'une tournée viennoise de la troupe du Deutsches Theater berlinois, Karl Kraus critique le jeu de l'acteur Emanuel Reicher qui se veut naturaliste, mais

15. Die Fackel, $\mathrm{n}^{\circ}$, fin avril 1899, p. 28. 
qui se réduit au manque d'humour et de personnalité justifiés par la recherche de la «vérité humaine» :

Emanuel Reicher, ein vielfach interessierter und auch literarisch bestrebter Herr, hat wiederholt zur Feder gegriffen, um die Doctrin des Naturalismus $z u$ vertheidigen. Er ist der typische moderne Schauspieler, der seine kleinen episodistischen Gaben an der Fiction der "Menschendarstellung" zugrunde gehen lässt. Mangel an Humor, Mangel an innerem Temperament befähigen ihn zur Ausrede der Lebensechtheit ${ }^{16}$.

La fin de l'article est sévère pour le naturalisme qui consiste pour les acteurs à se garder de faire du pathos et à se racler la gorge comme si personne ne les écoutait, et pour les décorateurs à veiller à l'installation de poignées de porte bien réelles:

Das Neue dieser Schauspielkunst schien mir vorwiegend darin zu liegen, dass, wo die Gefühle versagen, im richtigen Momente ein dem Leben abgelauschtes Räuspern eingelegt und dass inneres Leid wie Kopfschmerz ausgedrückt wird. Eine Kunst, die seelische Explosionen ängstlich vermeidet, weil sie deren nicht fähig ist... Solch eine Revolutionierung der Scene findet ihren Rückhalt in einer Theaterkritik, die alle Forderungen des Naturalismus erfüllt sieht, wenn der Decorateur für einen echten Plafond und für echte Thürklinken gesorgt hat ${ }^{17}$.

Pour Karl Kraus, le naturalisme dénie la différence fondamentale entre la réalité poétique de la scène et le jugement esthétique - l'autonomie de l'œuvre d'art - et s'applique à produire des «effets de réel» que Kraus assimile à un simple illusionnisme ${ }^{18}$. Il refuse la confusion entre l'art et la vie quotidienne qui paralyse l'imagination du spectateur d'une représentation de style naturaliste et qu'il trouve terriblement ennuyeux $^{19}$. Sous la plume de Karl Kraus, les réticences envers le naturalisme se transforment bientôt en une opposition entre la grande tradition de la comédie et de la tragédie qu'il place du côté du Burgtheater

16. «Zum Gastspiel des „Deutschen Theaters“», Die Fackel, nº 10, début juillet 1899, p. 1-20, citation p. 18.

17. Ibid., p. 20.

18. "Das ist die schlimmste Wirkung des Naturalismus, dass er die Perspective der Natur, die Perspective der Bühne - und die Perspective des Urtheils beseitigt hat » («Vom Wechselgastspiel », Die Fackel, no 43, début juin 1900, p. 16-25, citation p. 21).

19. Voir l'aphorisme dans Karl Kraus, Aphorismes. Dires et contre-dires, Pierre Deshusses (trad. et préf.), Paris, Payot et Rivages, 2011, p. 125: «Le naturalisme au théâtre fait sonner de vraies pendules. Voilà pourquoi le temps se traîne » («Der Naturalismus der Szene läßt wirkliche Uhren schlagen. Darum vergeht einem die Zeit so langsam », dans «Sprüche und Widersprüche », Die Fackel, $\mathrm{n}^{\circ}$ 272-273, 15 février 1909, p. 40-48, citation p. 45). 
et le nouveau naturalisme à la mode dans les théâtres berlinois. Max Reinhardt, en quittant Vienne pour entamer une brillante carrière dans le secteur de l'industrie du spectacle berlinoise, est aux yeux de Kraus le détestable promoteur d'un naturalisme adapté au goût du grand public.

En 1903, à l'occasion d'une tournée du Kleines Theater de Max Reinhardt qui présente à Vienne sa mise en scène de Nachtasyl (Les BasFonds) de Gorki, Karl Kraus, agacé par le compte rendu enthousiaste de Hermann Bahr qui a loué chez Reinhardt la Weiterentwicklung (développement) du style de Brahm, Kraus estime que Reinhardt se conforme platement au style du Deutsches Theater caractérisé par le manque de poésie: "la mise en scène exacte d'un milieu et l'emploi des acteurs sont faits de telle manière que l'aveuglement régnant puisse les prendre comme des caractères profonds. " Pour Kraus, il n'y a pas lieu d'écrire, comme le fait Hermann Bahr, «comme si Vienne était le plus misérables des villages de théâtre et comme si les vestiges de la splendeur du Burgtheater n'étaient pas toujours plus décoratifs que les édifices utilitaires érigés par la sobriété berlinoise ${ }^{20} »$.

Jusqu'aux années 1930, Karl Kraus ne cessera de critiquer les productions "post-naturalistes» de Max Reinhardt recherchant les effets spectaculaires produits par la technique en vue du succès commercial. Karl Kraus rassemble ses arguments à l'occasion d'une interview berlinoise de Hofmannsthal dans laquelle celui-ci affirme que le théâtre viennois n'a plus rien qui puisse être comparé à Brahm et à Reinhardt. Tout en condamnant les journalistes viennois qui portent aux nues des productions locales qu'il tient pour de la «cochonnerie patriotique» (vaterländische Schweinerei ${ }^{21}$ ), Kraus estime que «la virtuosité de l'art dramatique berlinois est basée [...] sur la technique d'une diction mal

20. «Eine exacte Milieuregie und Episodenspieler so verwendet, daß sie der Theaterblindheit als tiefe Charakteristiker erscheinen "; «als ob Wien das elendste Theaterdorf wäre und als ob nicht die Trümmer einer Burgtheaterherrlichkeit noch immer dekorativer wirkten als die von Berliner Nüchternheit aufgeführten Nutzbauten». Voir «Ein Anhänger der nördlichen Moderne» et "Der „Wertheim-Bazar“ des Herrn Reinhardt», dans Peter Sprengel et Gregor Streim, Berliner und Wiener Moderne. Vermittlungen und Abgrenzungen in Literatur, Theater, Publizistik, WienKöln-Weimar, Böhlau, 1998, p. 154-157 et p. 175-179. Citations de Hermann Bahr, "„Kleines Theater“ und „Nachtasyl“», dans Glossen. Zum Wiener Theater (1903-1906), Berlin, Fischer, 1907, p. 236-248, et de Karl Kraus, « [Zum Gastspiel des Kleinen Theaters] », Die Fackel, no 138, mi-mai 1903, p. 14-17, p. 176.

21. «Hugo v. Hofmannsthal hat es gewagt...», Die Fackel, nº 175, 17 février 1905, p. 17-22, citation p. 17. 
articulée 22 ». Cette idée que le naturalisme et sa descendance durant le premier tiers $\mathrm{du} \mathrm{xx}^{\mathrm{e}}$ siècle sont une manifestation de la décadence de la culture linguistique contemporaine sera désormais au centre de la critique krausienne du naturalisme et de ses épigones, comme par exemple dans l'aphorisme de juillet 1907 :

Mein Blick fiel auf die letzte Seite von Max Halbe's „Jugend“. Wie jung war damals die Literatur! Hänschen wirft sich über Annchens Leichnam mit dem Rufe : „A-us!“Stünde „Aus!“, hätte es der Darsteller nicht getroffen. In der Tat, der Naturalismus war der Schwimmmeister der Unzulänglichkeit. Wenn er ihr nicht den Gürtel des Dialekts gab, hielt er ihr mindestens mit solchen Anweisungen die Stange ${ }^{23}$.

La manière naturaliste n'a pas épargné l'opérette dont Karl Kraus déplore la déchéance dans l'industrie du spectacle de divertissement populaire:

Was mich an dem Enthusiasmus für die Operettenschande am tiefsten berührt, ist die demokratisierende Wirkung, die von ihr auszugehen scheint. Man gewahrt eine förmliche Lust, sich mit Helden und Schicksalen der neuen Operettenwelt zu encanaillieren [...] Und es ist dann nur natürlich, daß wir unser Interesse für die unvermummten Träger der Handlung auch auf ihr Leben außerhalb der Bühne erstrecken. Der Naturalismus des singenden Kommis erleichtert die Identifizierung mit der Privatperson des Darstellers, und unser Schauspielerkultus, der ehedem ein gerechter Lohn der künstlerischen Persönlichkeit war, ist bloß die Konsequenz einer einmal übernommenen gesellschaftlichen Verpflichtung ${ }^{24}$.

Ce procès du théâtre naturaliste qui, selon Kraus, représente sur la scène la réalité la plus vulgaire pour «faire vrai», aboutit au poème intitulé «Naturalismus», publié en décembre 1918 dans Worte in Versen IV :

Als anno neunzig war die Bühnenluft verstaubt, da hat die Polizei es ausdrücklich erlaubt, aufs Podium zu spucken.

Da fühlte die Natur sich ganz und gar erfrischt, so hat man in Berlin mal tüchtig aufgemischt, wer wollte da noch mucken.

Und daß am Ende nicht der alte Bühnenton entarten möchte in der trägen Tradition

22. «die Virtuosität der Berliner Theaterkunst basiert [...] auf der Technik der unartikulierten Sprache» (ibid., p. 21).

23. «Kehraus», Die Fackel, n 229, 2 juillet 1907, p. 1-17, citation p. 9 et suiv.

24. «Grimassen über Kultur und Bühne», Die Fackel, n² 270-271, 19 janvier 1909, p. 1-18, citation p. 14 . 
und in der Zucht verlottern,

so lernten alle, die zu sprechen nicht erlernt

und welche von Natur schon vom Beruf entfernt,

die rechte Kunst, zu stottern.

Das rülpste sich nun aus wie vor 'nem Schweinetrog,

allein verboten war allein der Monolog

in allen Dialekten.

Beiseite spucken statt beiseite sprechen schien

Errungenschaft zu sein, als damals in Berlin

sie die Natur entdeckten ${ }^{25}$.

Contre le «naturalisme» des grosses productions dans le style de Max Reinhardt, Kraus réclame un théâtre d'acteurs et de texte, mettant la diction au premier plan et capable de susciter l'émotion (souvent renforcée par un accompagnement musical au piano), de mettre en mouvement l'imagination des spectateurs, sans avoir à recourir aux «effets de réel» des décors et des accessoires. Ce théâtre idéal, Kraus en donnait le modèle dans son «Theater der Dichtung " que Georg Knepler décrit en ces termes :

Eine Illusionsbühne kam da nicht zustande. Da sprach eben Karl Kraus, da waren Tisch, Stuhl, Text, Licht, und die vorgelesenen Szenenbilder und Regieanweisungen bauten zwar eine Bühne vor den Hörern auf, aber eine, die die Phantasie zwar beflügelte, aber eine illusionsfreie ${ }^{26}$.

Le 21 novembre 1932, pour le soixante-dixième anniversaire de Gerhart Hauptmann (fêté en Allemagne en même temps que le centenaire de la mort de Goethe), Karl Kraus donne une lecture publique des Tisserands à Prague. Il ajoute ce commentaire dans Die Fackel :

Die Wiedergabe der „Weber" mit den Mitteln, über die das Theater der Dichtung verfügt, bezweckt als Ehrung des Dichters die Rehabilitierung des Werkes nach all dem, was die Berliner Bühnen durch all die Zeit mit ihm aufgeführt haben. Sie stellt, mit der Bewahrung, vielleicht auch Verstärkung, lebendigsten Erinnerns jeder einzelnen Stimme von damals, stilistisch die Gestalt wieder her jener Berliner Uraufführung vom 26. Februar 1893, die

25. «Naturalismus» («bisher ungedruckt», précise Kraus dans Die Fackel, n ${ }^{\circ}$ 508-513, avril 1919, p. 24, en annonçant la publication du $4{ }^{\mathrm{e}}$ vol. de ses Worte in Versen), dans Worte in Versen IV, Leipzig, Verlag der Schriften von Karl Kraus (Kurt Wolff), décembre 1918. Karl Kraus mettra ce poème au programme de sa lecture publique du 11 novembre 1924, à Vienne (voir Die Fackel, nº 668-675, décembre 1924, p. 52).

26. Georg Knepler, Karl Kraus liest Offenbach. Erinnerungen. Kommentare. Dokumentationen, Wien, Löcker, 1984, p. 14. 
(mit den Herren Rittner, Pauli, Fischer, Pagay, Thielscher, Nissen und Frau Bertens) den Gipfel eines schauspielerisch doch fundierten Bühnenrealismus bedeutet hat und eine Gesamtleistung, die von keinem Berliner Regisseur bei keiner Gelegenheit auch nur annähernd jemals wieder erreicht wurde ${ }^{27}$.

Ainsi, en 1932, Karl Kraus juge paradoxalement nécessaire de "réhabiliter»Les Tisserands que les mises en scène naturalistes berlinoises ont profané, déshonoré, de rendre à la pièce de Hauptmann tout son éclat originel, celui de la mise en scène de la première de février 1893 qui n'était pas encore gâtée par la manière naturaliste berlinoise mais donnait l'exemple d'un « réalisme bien conçu».

\section{Contre le «zolaïsme» et le parisianisme}

Les raisons de l'antipathie de Karl Kraus pour le naturalisme d'Émile Zola sont nombreuses. Aux yeux du directeur de Die Fackel, Zola est le symptôme du mal qui menace le plus la littérature contemporaine: le renoncement à l'autonomie esthétique de l'œuvre et la contamination de l'écriture littéraire par le journalisme ${ }^{28}$. Kraus ne croit pas à «l'hybridation» féconde du texte littéraire et des discours journalistiques $^{29}$. Il serait plutôt proche du point de vue de Balzac: «Il n'y a pas de livre dont la lecture soit possible après celle des journaux ${ }^{30}$ » (Le Voleur, 10 décembre 1830).

À ces considérations de principe qui font de Zola, aux yeux de Kraus, une des manifestations les plus spectaculaires - et les plus consternantes - de la dégradation de la littérature contemporaine, s'ajoute la méfiance de Karl Kraus envers les dreyfusards et le dreyfusisme et

27. «Vorlesung Prag 25. 11. 1932 », Die Fackel, $n^{\circ} 885-887$, fin décembre 1932, p. 16 et suiv., citation p. 17 .

28. Voir Pierre-Marc de Biasi, Gustave Flaubert. Une manière spéciale de vivre, Paris, Grasset, 2009, p. 272: «Quand Zola décide de se faire chercheur et journaliste, c'est pour devenir, avant la lettre, une sorte d'ethnologue de la société contemporaine, pour se doter de matériaux qui lui permettront de délivrer un message sur les grands problèmes de son temps. Ce n'est pas du tout l'objectif de Flaubert. »

29. Voir Marie-Ève Thérenty, "La littérature-journal», dans Dominique Kalifa, Philippe Régnier, Marie-Ėve Thérenty et Alain Vaillant (dir.), La civilisation du journal. Histoire culturelle et littéraire de la presse française au XIx ${ }^{e}$ siècle, Paris, Nouveau Monde, 2011, p. 1509-1521.

30. Cité par Judith Lyon-Caen, «Lecteurs et lectures: les usages de la presse au $\mathrm{XIX}^{\mathrm{e}}$ siècle», dans La Civilisation du journal, op. cit., p. 23-60, citation de Balzac p. 29. 
sa critique des usages et mésusages du nom de Zola dans la presse libérale viennoise. L'attitude de Kraus face à l'affaire Dreyfus apparaît aujourd'hui comme une de ses provocations les moins convaincantes ${ }^{31}$. Elle n'est compréhensible que si l'on admet que les déclarations et les opinions de Karl Kraus, pendant l'Affaire, sont inspirées par d'autres préoccupations que la recherche de la vérité et l'exigence de justice dans une cause qu'il considère comme étrangère et lointaine. Son hostilité à Theodor Herzl, un des champions viennois du dreyfusisme militant, et à la presse libérale qui donne le ton dans les milieux dirigeants à Vienne et à Berlin ont certainement pesé beaucoup plus lourd que l'examen des arguments pour et contre Dreyfus.

Karl Kraus a bien vu que, depuis le début de l'affaire Dreyfus, les journaux ont servi « de vecteur privilégié au développement du scandale, à sa mise en scène, mais aussi à la mobilisation de l'opinion par les dreyfusistes $^{32}$ ». Les interventions de Zola dans les journaux dreyfusards lui apparaissent comme une fatale erreur d'appréciation sur la presse qui, aux yeux de Kraus, est l'ennemie naturelle des intellectuels, non leur alliée, car « une presse soumise aux lois du marché a d'abord pour fonction de conforter les préjugés du public ${ }^{33}$ »: à Vienne, la presse nationale-allemande, cléricale et antisémite est antidreyfusarde, comme ses lecteurs le demandent, tandis que la presse libérale défend Dreyfus et célèbre le noble engagement de Zola, flattant le penchant de ses lecteurs pour les opinions conformes au "politiquement correct» de la bourgeoisie d'argent et de culture, en particulier de la bourgeoisie juive assimilée. Karl Kraus a devancé ceux, parmi les intellectuels, qui se sont rendu compte, au cours de l'affaire Dreyfus, « du risque que fait encourir à la démocratie un journalisme sans principes et sans déontologie»: « À partir de cette expérience douloureuse, certains essaient d'inventer de nouveaux relais pour combattre cette dépendance du débat public par rapport à ce média dominant ${ }^{34}$. » Zola, vu par Kraus, aura incarné la dangereuse illusion d'une alliance possible entre la presse et les intellectuels.

31. «Karl Kraus und die Dreyfus-Affäre », Études germaniques, $71^{\mathrm{e}}$ année, $\mathrm{n}^{\mathrm{o}}$ 3, 2016, juillet-septembre («Wien-Paris im Licht der Fackel von Karl Kraus »), p. 327-359.

32. Christophe Charle, Le siècle de la presse (1830-1939), Paris, Seuil, "L'Univers historique ", 2004, p. 201.

33. Ibid.

34. Ibid., p. 202. 
La seule occasion de parler en faveur d'Émile Zola est donnée à Karl Kraus par l'hommage publié en première page de la Neue Freie Presse le jour de la mort de Zola, le 29 septembre 1902. Kraus est indigné de voir l'éditorialiste du grand quotidien libéral viennois suggérer que Zola aurait racheté mainte page ordurière de son œuvre romanesque par le seul «J'accuse!» et il oppose à cet hommage ambigu sa propre déclaration: "Pour tous ceux qui aiment l'art, Zola est mort », ce qui ne veut pas forcément dire que Karl Kraus lui-même aime les romans de Zola :

„In seinen umständlichen, nicht immer kurzweiligen Romanen gab es einzelne Stellen von fragwürdiger Sauberkeit... Er wäre ein großer, ein unvergesslicher Mensch gewesen, auch wenn von seinem vielbändigen Werke nichts bliebe, als dieses einzige Blatt, auf dem ,J'accuse!" geschrieben stand." Liest man aus diesen Sätzen des Nachrufs, den die ,Neue Freie Presse'Zola gewidmet, nicht die Verlegenheit, ja den Widerwillen heraus, in einer Apotheose, die seinem „Kampf für die Wahrheit" gilt, auch einige Worte über den Dichter $z u$ sagen? „Er hat sich und sein Werk gereinigt durch die größte That seines arbeitsreichen Lebens" - wer verkennt in diesem Dictum Herrn Herzls Meinung, daß Zolas „Schmutz" einer Reinigungsthat von überwältigender Größe bedurfte, und wer zweifelt, den Abstand zwischen „Neuer Freier Presse“ und "Deutschem Volksblatt" in der Beurtheilung der "Affaire" ermessend, daß es sich lediglich um eine Nuance des literarischen Urtheils und des Tons handelt, wenn das "Deutsche Volksblatt" schreibt, Zola habe seine Feder „direct in die übelriechende Flüssigkeit der Gosse oder in die schmutzige Flut der Jauche gesenkt"? Völlig Gleiches denken die antisemitische und die semitische Journaille, indem sie Entgegengesetztes sagen [...].

Allen, die die Kunst lieben, ist Zola gestorben; und es ist ein tröstliches Bewusstsein, daß er nicht denen gelebt hat, die sein Tod nur vor die eine Frage stellte: Wird Dreyfus hinter dem Sarge gehen ${ }^{35}$ ?

L'animosité de Karl Kraus envers Theodor Herzl - dont le nom est évoqué, on l'a vu, dans cet article «Zola est mort, vive Dreyfus » - a certainement pesé dans son rejet de l'œuvre d'Émile Zola et du naturalisme parisien. Dans les nombreuses études consacrées à Herzl, les relations du correspondant de la Neuen Freien Presse à Paris d'octobre 1891 à juillet 1895 avec les naturalistes français ont rarement été abordées ${ }^{36}$. Or la pièce de Theodor Herzl, Das neue Ghetto (Le Nouveau Ghetto),

35. «Zola est mort, vive Dreyfus! », Die Fackel, $\mathrm{n}^{\circ}$ 117, 7 octobre 1902, p. 21-23, citation p. 22 et suiv.

36. Max Likin, "Rights of Man, Reason of State: Émile Zola and Theodor Herzl in Historical Perspective», Jewish Social Studies, vol. 8 (n. s.), $\mathrm{n}^{\mathrm{o}}$ 1, hiver 2001, p. 126-152 ne traite pas la question du point de l'histoire de la littérature. 
écrite en octobre 1894 à Paris, dont la première aura lieu en janvier 1898 à Vienne, au Carltheater, peut être considérée comme un drame social de style naturaliste ${ }^{37}$. L'influence de Germinal (1885) est, à mon avis, évidente dans les scènes du Nouveau Ghetto qui traitent de la misère des mineurs de Dubnitz, de leur grève et de l'accident survenu dans la mine dont la responsabilité est rejetée sur les grévistes.

Autant que la question sociale, c'est la « question juive» que Theodor Herzl met au centre de sa pièce: il veut montrer que l'antisémitisme contemporain qui vise les Juifs viennois culturellement assimilés et socialement intégrés crée un "nouveau ghetto », un ghetto dont l'enceinte est invisible, mais dont la réalité est aussi cruelle que celle du ghetto médiéval. Cette tentative de transposition du genre traditionnel de «l'histoire du ghetto" (Ghettogeschichte) dans le milieu de la grande ville est, chez Herzl, une sorte de réponse au souhait de Hermann Menkes qui, dans le journal hebdomadaire Die Zeit du 17 juillet 1897 , sous le titre "Ghetto-Naturalismus ${ }^{38}$ ", réclamait un renouvellement d'un genre illustré par Leopold Kompert, Aaron Bernstein et Leopold Sacher-Masoch ${ }^{39}$ (les auteurs de référence en matière de Ghettogeschichte cités par Hermann Menkes), mais devenu archaïsant à force d'idéalisation du ghetto des villages et des petites villes des marges orientales de l'Europe centrale, présenté désormais comme un conservatoire, pour ainsi dire, de la vie juive intacte et non dénaturée par la modernisation. Contre cette tendance à l'idéalisation du mode de vie rural et provincial des communautés juives de l'Est, Menkes appelait à un naturalisme capable de représenter la condition juive contemporaine,

37. Sur cette pièce de Herzl et les évocations de la représentation de janvier 1898, moqueuses comme on s'en doute, sous la plume Karl Kraus, voir Jacques Le Rider, Wien als „Das neue Ghetto"? Arthur Schnitzler und Theodor Herzl im Dialog, Wien, Picus, "Wiener Vorlesungen, 171 », 2014.

38. Hermann Menkes, "Ghetto-Naturalismus », Die Zeit, vol. 12, ${ }^{\circ}{ }_{146}, 17$ juillet 1897, p. 40-41. Cité d'après Gotthart Wunberg (éd.), Das Junge Wien. Österreichische Literatur- und Kunstkritik 1887-1902, Tübingen, Niemeyer, 1976, vol. 2, p. 758 (texte $\mathrm{n}^{\circ}$ 97.42).

39. Leopold von Sacher-Masoch déclarait pour sa part, en ennemi du naturalisme: "Naturalismus ist nicht von der Natur inspirirt worden wie jede wahre Kunst, sondern von der Wissenschaft, und deshalb hat er die Grenzen der Wissenschaft und Kunst niedergerissen und aus seinen Werken etwas gemacht, was weder Wissenschaft noch Dichtung ist " ( Die naturalistische Epidemie. Nach persönlichen Eindrücken », Die Gegenwart, vol. 35, n 25, 1889, p. 390-393, cité dans Manfred Brauneck et Christine Müller (éd.), Manifeste und Dokumente zur deutschen Literatur 1880-190o, Stuttgart, Metzler, 1987, p. 376). 
affectée par le nouvel antisémitisme sévissant dans toute l'Europe, en particulier dans les grandes villes, et citait un modèle à suivre : le récit de Jakob Elias Poritzky, Keinen Kadosch wird man sagen ${ }^{40}$.

Les hommages emphatiques rendus dans la presse libérale viennoise, détestée par Karl Kraus, à Émile Zola, le "zolaïsme» dreyfusard de Theodor Herzl et son "naturalisme de l'histoire du nouveau ghetto" dans la pièce Das neue Ghetto rendaient le naturalisme parisien difficilement supportable à Kraus. Mais une autre de ses bêtes noires le lui rendait encore plus haïssable: Hermann Bahr.

Celui-ci, après avoir fréquenté assidûment les cercles naturalistes pendant son séjour parisien, à partir de novembre 1888 , et tout en imitant L'Euvre (1886) de Zola dans son roman Die gute Schule (La Bonne École) de $1890^{41}$, amorçait, depuis son article «Die neue Psychologie» de septembre 1890, où il affirmait: "Après l'épisode physique du naturalisme, nous avons besoin de psychologie ${ }^{42}$ ", son "dépassement du naturalisme ». Ce soi-disant dépassement, aux yeux de Karl Kraus, n'était que la gesticulation médiatique d'un journaliste cherchant à donner à son éclectisme touche-à-tout en quête du dernier cri à la mode - parfaite incarnation du modernisme rejeté par l'antimoderne Kraus - l'apparence d'une démarche esthétique mûrement réfléchie et le ton d'un chef de file autoproclamé. Le dépassement du naturalisme prôné par Hermann Bahr à partir de 1891 est en réalité sélectif, de sorte que son "post-naturalisme» tient sur certains points d'un naturalisme tardif, comme le fait remarquer Norbert Bachleitner :

Seinem ästhetischen Programm werden von nun an kaum noch neue Elemente hinzugefügt. In dem Aufsatz „Vom jüngsten Frankreich“ (1891) wird bereits eine gewisse Orientierungslosigkeit bei dem literarischen Reporter sichtbar. [...] Bahrs Projekt der Überwindung des Naturalismus, kann man [...] als eine Synthese von Zola und Bourget betrachten ${ }^{43}$.

40. Jakob Elias Poritzky, „Keinen Kadosch wird man sagen... “Aus dem Tagebuch eines Verwaisten, Leipzig, Reclam, «Universal-Bibliothek, nº 3568 », 1897.

41. Hermann Bahr, Die gute Schule, Berlin, Fischer, 1890.

42. «Psychologie tut uns Noth [nach der] physikalischen Episode des Naturalismus» (Hermann Bahr, "Die neue Psychologie», dans Moderne Dichtung, aoûtseptembre 1890 , cité d'après Das Junge Wien, op. cit., p. 93, texte ${ }^{\circ}$ 90.24).

43. Norbert Bachleitner, "Hermann Bahr und die französische Literatur", dans Hermann Bahr. Mittler der europäischen Moderne, Hermann Bahr-Symposion, Linz, Adalbert-Stifter-Inst. des Landes Oberösterreich, 1998, p. 145-160, citation p. 158 et suiv. 
Ainsi l'ancien disciple de la "bonne école» naturaliste parisienne ne se détache pas entièrement de ses premiers maîtres. Son dépassement (Überwindung) est une Aufhebung qui dépasse tout en conservant. La distinction entre le naturalisme au théâtre et le naturalisme dans le roman permet même à Hermann Bahr de suggérer qu'il restera un auteur dramatique naturaliste tout en devenant un romancier post-naturaliste :

Vom Bühnennaturalismus will ich reden, nur von diesem. Der Buchnaturalismus, des Romans und der Novelle, gehört schon wieder der Geschichte. Sein Kampf, sein Sieg, seine Überwindung liegen hinter uns [...]. Sein erledigtes Schicksal werden nun wohl die Professoren in ihre Vorlesungen setzen. [...] Also vom Theater will ich reden, auf welchem der Naturalismus noch erst in den Anfängen ist. Hier möchte ich constatiren, dass es Naturalismus und Naturalismus gibt [...]. Außer dem Namen haben der französische Bühnennaturalismus und der deutsche wenig gemein. [...] Der Erfolg des französischen Bühnennaturalismus ist der Skandal, der Erfolg des deutschen ist die Langeweile ${ }^{44}$.

Ces jugements à l'emporte-pièce ont dû irriter le jeune Karl Kraus pour au moins deux raisons: d'abord parce que Bahr inclut Gerhart Hauptmann, dont Kraus est un admirateur, dans le "naturalisme ennuyeux» propre aux Allemands et se pose implicitement en représentant d'une «troisième voie» viennoise qui permettrait de sortir de l'alternative théâtre naturaliste à scandale en France, théâtre naturaliste soporifique en Allemagne. Ensuite parce que Karl Kraus, lui aussi, tend à admettre cette distinction entre naturalisme dans le roman et naturalisme au théâtre, à rejeter le premier et à préférer le second, mais ne peut pas admettre que les goûts de Hermann Bahr ressemblent aux siens.

La sensibilité esthétique de Karl Kraus a été formée à l'école du Burgtheater et des théâtres plus populaires qui lui ont fait découvrir, dans sa jeunesse, les comédies de Nestroy et Raimund et les opérettes d'Offenbach. En revanche, le roman contemporain ne semble pas l'avoir

44. Hermann Bahr, «Naturalismus und Naturalismus », Die Gegenwart. Wochenschrift für Literatur, Kunst und öffentliches Leben, vol. $38, \mathrm{n}^{\circ} 37,13$ septembre $1890, \mathrm{p} .170$ et suiv., cité d'après Das Junge Wien, op. cit., p. 111-114, texte $\mathrm{n}^{\circ}$ 90.29), citation p. 111. Dans la suite de cet article, Bahr compare d'abord Les Chapons (1890) de Lucien Descaves et Georges Darien avec Familie Selicke (1890) d'Arno Holz et Johannes Schlaf, puis Das Friedensfest (1890) de Gerhart Hauptmann avec Monsieur Betsy de Paul Alexis et Oscar Méténier (1890). 
jamais autant captivé ${ }^{45}$. Ses aveux sur ce point ne laissent guère de place au doute :

Einen Roman zu schreiben, mag ein reines Vergnügen sein. Nicht ohne Schwierigkeit ist es bereits, einen Roman zu erleben. Aber einen Roman zu lesen, davor hüte ich mich, so gut es irgend geht. Wo nehme ich nur all die Zeit her, so viel nicht $z$ u lesen ${ }^{46}$ ?

Le témoignage d'Elias Canetti le confirme: «Les romanciers, les narrateurs en général, il ne s'en mêlait pas; je crois qu'ils ne l'intéressaient pas beaucoup ${ }^{47}$." On peut voir dans cette préférence de Karl Kraus pour le théâtre une des raisons de son manque d'intérêt pour le naturalisme français qui s'est illustré surtout dans le domaine du roman. Le fait que Kraus ait fait l'éloge du roman d'Otto Stoessl Sonjas letzter Name $(1908)^{48}$, déclarant que «[p]our recommander ce livre, je me

45. Précisons que Karl Kraus a apprécié Heinrich Mann et Dostoïevski, mais aussi le Malte Laurids Brigge de Rilke (voir Berthold Viertel, «Rilkes Buch», Die Fackel, $\mathrm{n}^{\mathrm{o}}$ 309-310, 31 octobre 1910, p. 20-24). Pendant la guerre, il fait l'éloge de la prose narrative de Stifter. On peut noter qu'en 1925 il parle de ses articles dans Die Fackel comme de chapitres d'un cycle romanesque: "Personen, die ich in szenische Gestalten und in die Romanfiguren meiner Glossenwelt transformiert habe, treten nach dem ersten Chok frohgemut aus dem Satzbau, in den ich sie einfing und der doch zumeist ihr eigener war " ("Zweihundert Vorlesungen und das geistige Wien », Die Fackel, n ${ }^{\circ} 676-678$, janvier 1925, p. 47-68, citation p. 51 et suiv.).

46. Karl Kraus, Sprüche und Widersprüche, V : Der Künstler, dans Aphorismen, Schriften, op. cit., p. 119. $1^{\text {re }}$ publication dans «Tagebuch », Die Fackel, n ${ }^{\circ}$ 259-260, 13 juillet 1908, p. 35-56, citation p. 47 (sous la forme de 2 aphorismes successifs; le ${ }_{1}{ }^{\mathrm{er}}$ : «Wo nehme ich nur all die Zeit her... »; le $2^{\mathrm{e}}$ : "Einen Roman zu schreiben... », etc.). Traduction: Aphorismes. Dires et contre-dires, Pierre Deshusses (trad.), op. cit., p. 145.

47. Elias Canetti, «Karl Kraus. École de la résistance», dans La Conscience des mots, Roger Lewinter (trad.), Paris, Albin Michel, 1984, p. 51-64, citation p. 62.

48. Otto Stoessl, Sonjas letzter Name. Eine Schelmengeschichte, München, Georg Muller, 1908. Karl Kraus confie à Samuel Lublinski le soin de présenter le romancier Otto Stoessl: «Der Erzähler Otto Stoessl», Die Fackel, no 309-310, 31 octobre 1910, p. 7-15, où sont commentés Egon und Danitza, Sonjas letzter Name et Negerkönigs Tochter. Dans cet article de Lublinski, on remarque particulièrement le passage où il est question, à propos de Sonjas letzter Name, de la représentation de l'Autriche contemporaine: "Dieses seltsame Reich ist gegenwärtig im eben entwickelten Sinn ein schlechthin episches Reich. Noch ist die stramme moderne Disziplinierung nicht so weit vorgeschritten, daß dem Hochstapler jede Hoffnung benommen wäre, zu einer anständigen sozialen Stellung zu gelangen [...]. Dazu kommt die bunte Ethnographie des Landes, die Fülle der nationalen Typen, die schwankende Unsicherheit der staatlichen Verhältnisse seit dem Sturz des Zentralismus, und endlich, im Gegensatz dazu, so viel fast kleinbürgerlich pathetische und doch solide Ideologie» (p. 12). 
contenterai de dire que j'ai lu jusqu'au bout ${ }^{49}$ ", ne peut pas servir ici de contre-argument, puisque cette œuvre d'Otto Stoessl a pour intention de renouveler le genre du roman picaresque dans l'esprit du néoclassicisme.

\section{En deçà et au-delà du naturalisme : Frank Wedekind}

Karl Kraus admirait Frank Wedekind, auteur le plus souvent rattaché au naturalisme, autant que Gerhart Hauptmann, mais il noua avec l'auteur de La Boîte de Pandore des relations d'amitié qu'il n'eut jamais avec Hauptmann ${ }^{50}$. Ce qui frappe dans la conférence d'introduction à la représentation privée de Büchse der Pandora organisée par Karl Kraus le 29 mai 1905, c'est que Kraus tient à placer Wedekind, comme il le fait constamment à propos du Hauptmann de la première période, au-dessus du mouvement naturaliste :

Daß Frank Wedekind ein Menschenschilderer ist, wäre schon ein Lob, das ihn über die Milieuschilderer himmelhoch emporhöbe. Aber er ist auch der erste deutsche Dramatiker, der wieder dem Gedanken den langentbehrten Zutritt auf das Theater verschafft hat. Alle Natürlichkeitsschrullen sind wie weggeblasen. Was in und hinter den Menschen liegt, ist wieder wichtiger, als was für einen Sprachfehler sie haben ${ }^{51}$.

À la platitude des naturalistes qui confondent la littérature et le reportage social, accréditant l'idée d'un déterminisme du milieu à la façon d'Hippolyte Taine et caractérisant leurs personnages par leur langue argotique (rendue de manière plus ou moins conventionnelle), Karl Kraus oppose la «vérité » et «le naturel supérieur» (höhere Natürlichkeit ${ }^{52}$ ) des personnages de Wedekind et de leur langage qui font de cet auteur un nouveau Shakespeare ${ }^{53}$. "Naturalisme », sous la plume de Kraus, est devenu un mot repoussoir avec lequel ses auteurs préférés, le Gerhart Hauptmann des Tisserands et le Frank Wedekind de La Boîte de Pandore, n'ont en réalité rien à voir.

49. "Zu dessen Empfehlung ich nicht mehr sagen kann, als dass ich ihn ausgelesen habe ", Die Fackel, no 275-276, 22 mars 1909, p. 18.

50. Voir Karl Kraus-Frank Wedekind, Briefwechsel 1903 bis 1917, Mirko Nottscheid (éd.), Würzburg, Königshausen \& Neumann, «Wedekind-Lektüren, 5 », 2008.

51. Karl Kraus, "„Die Büchse der Pandora“, Einleitung zur Wiener Aufführung am 29. Mai $1905 »$, Die Fackel, n ${ }^{0}$ 182, 9 juin 1905, p. 1-14, citation p. 7.

52. Ibid.

53. «Hier, besonders im dritten Akt, hat die Hand eines neuen Shakespeare den tiefsten Griff in das Menscheninnerste getan», ibid., p. 8. 